\title{
Período crítico de competencia de la Zanahoria (Daucus carota L.) y malezas asociadas al cultivo
}

\author{
Critical competition period between Carrot (Daucus carota L.) and \\ weeds associated to the crop
}

Mónica Yadira Dotor R. ${ }^{\text {; }}$ Leonardo Ariel González M. ${ }^{\text {; }}$ Ana Cruz Morillo C. ${ }^{3}$

1 Ingeniero Agrónomo, M.Sc., Universidad Nacional de Colombia, Bogotá, Colombia, mydotorr@unal.edu.co.

2 Ingeniero Agrónomo, M.Sc., Universidad Pedagógica y Tecnológica de Colombia, Tunja, Colombia, leonardo. gonzalez@uptc.edu.co.

3 Ingeniero Agrónomo, Ph.D., Universidad Pedagógica y Tecnológica de Colombia, Tunja, Colombia, ana.morillo@ uptc.edu.co.

Citar: Dotor, M., González, L. \& Morillo, A. (2018). Período crítico de competencia entre zanahoria (Daucus carota L.) y malezas asociadas al cultivo. Rev. Cienc. Agr. 35(1): 5-15. doi: http://dx.doi.org/10.22267/ rcia.183501.78.

Recibido: Mayo 01 de 2017. $\quad$ Aceptado: Octubre 18 de 2017.

\section{RESUMEN}

Las malezas constituyen uno de los problemas fitosanitarios que afectan la rentabilidad de los cultivos. El periodo crítico de competencia con el cultivo (PCC), permite prever el nivel de interferencia de las malezas sobre la producción del cultivo de interés. El objetivo de esta investigación fue determinar el PCC entre zanahoria y las plantas asociadas al cultivo, en el municipio de Ventaquemada, departamento de Boyacá, implementando un diseño en Bloques Completos al Azar, con 12 tratamientos y cuatro repeticiones, el cual incluía un grupo de tratamientos libres de malezas por un periodo de tiempo (10, 20, 30, 40 ó 50 días), al término del cual se permitía el crecimiento de las mismas en el cultivo, de modo que el cultivo estuviese primero libre y luego en competencia (LC) y en el segundo grupo de tratamientos se permitía primero el desarrollo del proceso de competencia entre la maleza y el cultivo por un intervalo de tiempo igual al del grupo anterior y luego se retiraban las malezas. El mayor número de plantas cosechadas se obtuvo en la parcela libre de competencia durante los primeros 50 días después de emergencia. El mayor rendimiento se obtuvo en el tratamiento que se mantuvo limpio 
durante todo el ciclo de cultivo, con una producción de $5810 \mathrm{~g} \mathrm{~m}-2$, superior en 2252g a la obtenida en el punto crítico de competencia. para las condiciones evaluadas, el periodo crítico de competencia en zanahoria, se sitúa entre los 20 a 45 días, después de la emergencia del cultivo.

Palabras clave: Densidad, dinámica de poblaciones, monitoreo, diversidad.

\begin{abstract}
Weeds are one of the phytosanitary problems that affect crop's productivity. The critical period of competition (PCC) allows to predict the level of interference of weeds on the crop. The objective of this investigation was to determine the PCC between carrot and weeds or other accompanying plants, in the municipality of Ventaquemada, department of Boyacá. A completely randomized blocks, with 12 treatments and four repetitions, was used. It included a group of treatments free of weeds for 10,20, 30, 40 and 50 days, at the end each the weeds growth was allowed, so that the crop was first free and then in competition (FC). The second group of treatments allowed the initial competition between weeds and the crop, for the same period of first group at the end of each time period the weeds were removed. The largest amount of carrots plants was obtained in the free weeds plot during 50 days after emergence. The highest yield was attained in the free weeds treatment throughout the crop cycle $5810 \mathrm{~g}$ $\mathrm{m}-2$ ). It was $2252 \mathrm{~g}$ higher than the production obtained at the critical point of competition. In general, the critical period of competition in carrot was thorough 20 to 45 days after crop emergence.
\end{abstract}

Key words: Density, population dynamics, monitoring, diversity.

\section{INTRODUCCIÓN}

El manejo apropiado de la competencia interespecífica en el sistema productivo de Zanahoria (Daucus carota L.) constituye un factor determinante en el rendimiento y éxito comercial del cultivo. Como es común, a la par del cultivo, se encuentran especies competidoras, las cuales interfieren con el crecimiento y desarrollo de la especie cultivada, por ello, es necesario conocer el impacto de éstas en el cultivo, información que contribuye a obtener mejores rendimientos en la actividad productiva (Délye et al., 2013).

Uno de los aspectos importantes para tener un manejo integrado de las malezas, consiste en determinar el periodo crítico de competencia (PCC), que hace referencia al momento o época, en la cual es necesario realizar el control de malezas para evitar pérdidas económicas en el cultivo (Gantoli et al., 2013; Walsh et al., 2013; Knezevic y Datta, 2015; Korres y Norsworthy, 2015). El PCC puede ser establecido a través del registro en días calendario o en grados calor día acumulados. Esto permite calcular el tiempo en el cual se genera la máxima interferencia de las plantas nocivas con el cultivo, el cual puede variar para una misma especie, dependiendo de las características del agro-ecosistema en estudio, mediadas por las condiciones agroecológicas de la región, la altitud, el material genético, la población sembrada, la composición de las plantas competidoras, su densidad, distribución y el tiempo en el que compiten las plantas (Aramendiz et al., 2010). 
Debido a esto, algunas investigaciones difieren en los resultados del PCC, como la reportada en trigo por Hosseini et al. (2014), quienes ubican el período crítico de competencia entre 30 y 50 días después de la siembra, mientras que Villegas et al. (2004) en baja California, la sitúan entre 55 y 60 días después de la emergencia.

La evaluación del PCC se enmarca en un proceso de competencia interespecifica, en la cual se presentan dos condiciones diferentes, producto de las interacciones entre el cultivo y las plantas maleza. En primera instancia, el momento en que se presenta la mayor densidad de malezas por unidad de área, representa el tiempo en el cual las plantas pueden permanecer en el cultivo antes de empezar a competir de manera relevante con éste y en segundo lugar, el periodo mínimo, donde el cultivo debe permanecer libre de malezas, para que la presencia de estas plantas no genere un detrimento en la producción (Radosevich et al., 2007).

La evaluación de estas dos condiciones es la que ha permitido determinar periodos críticos para el control de malezas en los cultivos, dado que los experimentos que se realizan buscan cruzar los dos momentos para ubicar la mejor época de control de las malezas y permitieron la generación de modelos teóricos de control de las mismas (Knezevic y Datta, 2015).

La región del municipio de Ventaquemada, Boyacá, es reconocida por ser productora de hortalizas. Ahí se ha establecido el cultivo de zanahoria, el cual ha encontrado una buena adaptación, debido a las condiciones agroecológicas de la región. Sin embargo, la información del efecto de la interferencia de las especies acompañantes sobre el cultivo, es limitada. No se reportan estudios locales, que referencien el manejo apropiado y el efecto de la biodiversidad vegetal del agro-ecosistema sobre el cultivo de zanahoria.

Es a partir de la ausencia de trabajos de investigación en PCC de zanahoria, que se planteó la ejecución de ésta investigación, con el fin de obtener información básica y apropiada para el manejo de las plantas nocivas asociadas al cultivo. Se llevó a cabo un experimento que tuvo como objetivo establecer el periodo crítico de interferencia entre las malezas y el cultivo de zanahoria y determina el efecto de ésta competencia sobre el rendimiento de ésta especie hortícola.

\section{MATERIALES Y MÉTODOS}

El experimento se realizó en condiciones de campo, en la vereda Bojirque, del municipio de Ventaquemada, departamento de Boyacá latitud 5.417 y con $73.55^{\circ} 25^{\prime} 1^{\prime \prime} \mathrm{LN}, 73^{\circ} 30^{\prime} 0^{\prime \prime} \mathrm{LO}$, la cual está ubicada a $2.900 \mathrm{msnm}$, con una temperatura promedio de $13^{\circ} \mathrm{C}$ y un régimen de lluvias bimodal, $1367 \mathrm{~mm}$, zona calificada según Holdridge como Bosque Húmedo Montano (Holdridge, 2000). La época de establecimiento de los ensayos se ubicó entre mayo y noviembre de 2015. La investigación consistió en realizar un ensayo que permitiera establecer el periodo crítico de competencia entre las malezas y el cultivo de zanahoria, en condiciones de campo. El criterio de selección del lote fue que éste tuviese una trayectoria en producción de hortalizas y su historial de infestación de malezas se considera normal en la zona. La caracterización fisicoquímica del suelo seleccionado fue determinada a partir de una muestra del predio en estudio (Tabla 1). 
Dotor et al. - Competencia zanahoria (Daucus carota L.) y malezas.

Tabla 1. Caracterización fisicoquímica del suelo objeto de estudio.

\begin{tabular}{llll}
\hline Factor & Valor & Factor & Valor \\
\hline $\mathrm{pH}$ & 4,65 (fuertemente ácido) & $\mathrm{K}$ & $3,24 \mathrm{me} \cdot 100^{-1} \mathrm{~g}$ (alto) \\
C.E. & $0,17 \mathrm{mhols}$ (bajo) & $\mathrm{Mg}$ & $1,43 \mathrm{me} \cdot 100^{-1} \mathrm{~g}$ (medio) \\
Acidez & 0,7 (baja) & $\mathrm{Zn}$ & $2,69 \mathrm{ppm}$ (bajo) \\
$\mathrm{Al}$ & $0,5 \mathrm{me} .100^{-1} \mathrm{~g}$ (bajo) & $\mathrm{Na}$ & $0,22 \mathrm{me} .100^{-1} \mathrm{~g}$ (medio-alto) \\
$\mathrm{P}$ & $22,61 \mathrm{ppm}$ & $\mathrm{Fe}$ & $104 \mathrm{me} \cdot 100^{-1} \mathrm{~g}$ (alto) \\
$\mathrm{M} .0$. & $18,86 \%$ & $\mathrm{Mn}$ & $10,2 \mathrm{ppm}$ (bajo) \\
$\mathrm{Ca}$ & $11,02 \mathrm{me} .100^{-1} \mathrm{~g}$ (alto) & $\mathrm{Cu}$ & $0,19 \mathrm{pm} \mathrm{(bajo)}$ \\
\hline
\end{tabular}

La preparación del suelo se realizó, con dos pases de rastrillo y un pase de rotovator, de modo que se obtuviera una condición de suelo apropiado para la siembra del cultivo. El lote se fraccionó en melgas de $1,70 \mathrm{~m}$ de ancho por $100 \mathrm{~m}$ de largo. La semilla utilizada fue zanahoria híbrida TI-247 tipo Nantes, la cual se sembró con una densidad de 750.000 semillas ha- $^{-1}$. En pre-siembra se aplicó $300 \mathrm{~kg} \mathrm{ha}^{-1}$ de abono orgánico. A los 30 días de emergencia del cultivo, se aplicaron $200 \mathrm{~kg} \mathrm{ha}^{-1}$ de fertilizante grado 13-26-6 y tres meses después de la emergencia de la plántula, se aplicaron $150 \mathrm{~kg} \mathrm{ha}^{-1}$ de nitrato de calcio granulado. Las labores agronómicas de mantenimiento se efectuaron de acuerdo con las necesidades técnicas del cultivo (Gaviola, 2013).

Los tratamientos se ubicaron según el método aditivo (Villegas et al., 2004), bajo un diseño básico de Bloques Completos al Azar con 12 tratamientos y cuatro bloques. Como factor de bloqueo se estableció el diferencial de pendiente del lote en el cual se ejecutó el experimento.

Dos clases de tratamientos fueron utilizados: el primer tipo fue de tratamientos Libres de Competencia (LC) desde la siembra, durante un intervalo de tiempo determinado; este grupo estuvo constituido por 6 tratamientos, los primeros cinco tratamientos estuvieron libres de malezas 10, 20, 30, 40, 50 días después de siembra (dds) y el sexto tratamiento estuvo libre de malezas durante todo el ciclo de cultivo (Tabla 2). La segunda clase consistió de cinco tratamientos que se mantuvieron con malezas en un intervalo determinado de tiempo $(10,20,30,40$ y 50 días después de siembra) y un sexto tratamiento que se mantuvo con malezas durante todo el ciclo de cultivo (CL) (Tabla 2). La deshierba en cada uno de los tratamientos se realizó de manera manual.

Tabla 2. Tratamientos por cada clase aplicada.

\begin{tabular}{ll}
\hline Tratamientos (Clase 1: LC) & Tratamientos (Clase 2:CL) \\
\hline $\mathrm{LC}_{10}$ libre de malezas los primeros 10 dds & $\mathrm{CL}_{10}$ enmalezado los primeros 10 días dds \\
$\mathrm{LC}_{20}$ libre de malezas los primeros 20 dds & $\mathrm{CL}_{20}$ enmalezado los primeros 20 días dds \\
$\mathrm{LC}_{30}$ libre de malezas los primeros 30 dds & $\mathrm{CL}_{30}$ enmalezado los primeros 30 días dds \\
$\mathrm{LC}_{40}$ libre de malezas los primeros 40 dds & $\mathrm{CL}_{40}$ enmalezado los primeros 40 días dds \\
LC $_{50}$ libre de malezas los primeros 50 dds & $\mathrm{CL}_{50}$ enmalezado los primeros 50 días dds \\
LCT libre de maleza todo el ciclo de cultivo & $\mathrm{CTC}=$ Testigo(competencia todo el ciclo) \\
\hline
\end{tabular}

LC: Tratamientos inicialmente libres de malezas después en competencia; CL. Tratamientos inicialmente con malezas y luego limpios. 
Cada unidad experimental estuvo constituida por parcelas de $6 \mathrm{~m}^{2}$, para un total de $288 \mathrm{~m}^{2}$ efectivos. Los datos recolectados se tomaron de la parte central de las parcelas experimentales, utilizando un cuadro de $1 \mathrm{~m}^{2}$. Una vez recolectado el material, tanto de zanahoria como de malezas, fueron llevados a las instalaciones del Laboratorio de Control Biológico de la Universidad Pedagógica y Tecnológica de Tunja (UPTC) para realizar las diferentes mediciones.

Las variables a evaluar para los dos componentes del estudio fueron: 1) para las plantas male$z a$, se evaluaron las plantas presentes en cada tratamiento y peso fresco total de la biomasa aérea de las mismas en cada deshierba y 2) para el cultivo, número de plantas de zanahoria al final del ciclo del cultivo, la producción total de biomasa, la producción de raíces de valor comercial y la determinación del rendimiento agronómico.

Para determinar el rendimiento, se tomaron las raíces cosechadas, se seleccionaron y clasificaron por calidad, según los criterios del mercado de la hortaliza, en los que van discriminadas raíces de extra y primera, conformadas por zanahoria gruesa y bien formada, con un diámetro mínimo de $20 \mathrm{~mm}$ y $60 \mathrm{~mm}$ de longitud. Zanahoria de segunda, constituida por raíces no aptas, para incluirse en la categoría primera o extra y; raíces de descarte, incluye zanahorias bifurcadas, blandas, o de consistencia leñosa (ICONTEC, 1994). El análisis estadístico se ejecutó en el programa estadístico SPSS, evaluándose la normalidad, homocedasticidad, el Análisis de Varianza y la determinación de las diferencias estadísticas entre medios de tratamiento se realizó mediante la prueba de Tukey $(\alpha=0,05)$.

\section{RESULTADOS Y DISCUSIÓN}

Especies nocivas presentes. La presencia y comparación poblacional de las especies de malezas y la producción de biomasa aérea de estas durante el ensayo se observa en la Tabla 3. La especie que mostró una mayor producción de biomasa junto con una mayor frecuencia de aparición en todos los tratamientos fue la golondrina (Drymaria cordata L.) (Nono et al., 2014) y su propagación puede ser por tallos o por semilla. Esta especie se mostró con mayor abundancia en los tratamientos que se mantuvieron libres de competencia en las fases iniciales del cultivo y luego en competencia (tratamientos $\mathrm{LC}_{10}, \mathrm{LC}_{40}$ y el $\left.\mathrm{LC}_{50}\right)$.

La siguiente especie de mayor frecuencia fue trébol (Trifolium repens L.) (Nichols et al., 2014), especialmente en los tratamientos que se mantuvieron enmalezados durante las primeras fases del cultivo, con una producción máxima acumulada de $940 \mathrm{~g} \cdot \mathrm{m}^{-2}$ obtenida en el tratamiento $\mathrm{CL}_{10}$. Esta especie se presentó con menor abundancia en los tratamientos libres de competencia los primeros días y en competencia después y observándose ausente en el tratamiento $\mathrm{LC}_{20}$. Otras especies encontradas, aunque con menor densidad, pero que conservan interés debido a su presencia recurrente en los cultivos de zanahoria de la zona de estudio, fueron acedera $(R u-$ mex acetosella L.) (Yazdi et al., 2013), cerrajilla (Senecio vulgaris L.) (Cheng and $\mathrm{Xu}, 2015$ ), cilantrillo (Spergula arvensis L.) (Landesmann et al., 2013) y gualola (Polygonum nepalense Meisn.) (Nkuété et al., 2015). En el caso de S. vulgaris estuvo ausente en el tratamiento $\mathrm{LC}_{20}$. La mayor presencia de ésta maleza se encontró en el tratamiento $\mathrm{LC}_{10}$, seguido de los tratamientos $\mathrm{LC}_{30}$ $\mathrm{y} \mathrm{LC}_{50}$. 
Tabla 3. Producción de biomasa (g) de cada una de las especies de plantas nocivas encontradas en los diferentes tratamientos.

\begin{tabular}{|c|c|c|c|c|c|c|c|c|c|c|c|c|c|c|c|}
\hline 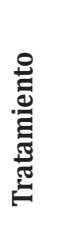 & 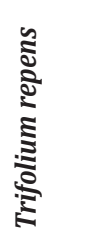 & 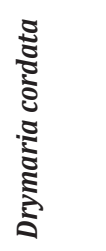 & 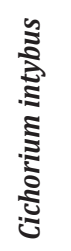 & 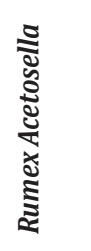 & 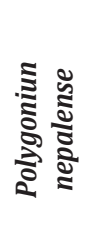 & 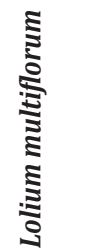 & 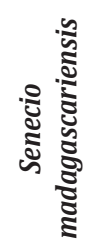 & 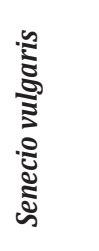 & 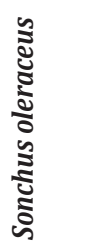 & 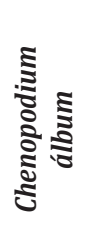 & 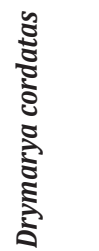 & 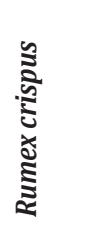 & 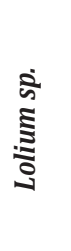 & 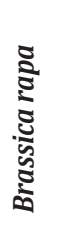 & 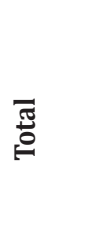 \\
\hline $\mathrm{L}_{10}$ & 940 & 285 & 230 & 91,25 & 540 & 75 & 40 & 25 & 20 & 0 & 0 & 0 & 0 & 0 & 1.706 \\
\hline $\mathrm{CL}_{20}$ & 832,5 & 182,5 & 0 & 205 & 332,5 & 222,5 & 20 & 75 & 0 & 20 & 226,3 & 35 & 0 & 0 & 1.889 \\
\hline $\mathrm{CL}_{30}$ & 647,5 & 537,5 & 0 & 170 & 147,5 & 118,8 & 20 & 75 & 0 & 37,5 & 0 & 195 & 0 & 0 & 1.801 \\
\hline $\mathrm{CL}_{40}$ & 200 & 452,5 & 80 & 282,5 & 20 & 62,5 & 40 & 12,5 & 20 & 37,5 & 0 & 31,25 & 0 & 0 & 1.389 \\
\hline$\underline{\mathrm{CL}}_{\underline{50}}$ & 162,5 & 7,5 & 100 & 302,5 & 62,5 & 0 & 7,5 & 125 & 0 & 57,5 & 0 & 0 & 120 & 0 & 883 \\
\hline$\underline{\mathrm{CTC}}$ & 187,5 & 427,5 & 0 & 50 & 57,5 & 105 & 17,5 & 75 & 280 & 0 & 0 & 45 & 125 & 0 & 1318 \\
\hline$\underline{\mathrm{LC}}_{10}$ & 137,5 & 807,5 & 0 & 25 & 87,5 & 162,5 & 30 & 565 & 187,5 & 0 & 30 & 635 & 0 & 0 & 2.635 \\
\hline $\mathrm{LC}_{20}$ & 0 & 345 & 0 & 387,5 & 10 & 0 & 45 & 12,5 & 56,25 & 0 & 0 & 0 & 0 & 162,5 & 1.009 \\
\hline $\mathrm{LC}_{30}$ & 307,5 & 350 & 0 & 340 & 107,5 & 202,5 & 25 & 242,5 & 537,5 & 0 & 0 & 362,5 & 0 & 0 & 2.675 \\
\hline $\mathrm{LC}_{40}$ & 230 & 723,75 & 0 & 192,5 & 0 & 70 & 7,5 & 50 & 531,25 & 20 & 0 & 0 & 0 & 0 & 1.825 \\
\hline $\mathrm{LC}_{50}$ & 380 & 586,25 & 30 & 385 & 0 & 0 & 42,5 & 275 & 175 & 0 & 0 & 243,8 & 0 & 0 & 2.118 \\
\hline
\end{tabular}

Por su parte, $R$. acetosella L. no se encontró en el tratamiento $\mathrm{LC}_{10}$ y fue muy abundante en los tratamientos $\mathrm{LC}_{20}, \mathrm{CL}_{40} \mathrm{y} \mathrm{CL}_{50}$ S. arvensis L. se observó en todos los tratamientos y su producción de biomasa fue una de las más bajas registradas, ya que en todos los casos fue inferior a los $50 \mathrm{~g} \mathrm{~m}^{-2}$. $P$. nepalense se encontró con una alta incidencia en los tratamientos $\mathrm{CL}_{10}$ y $\mathrm{CL}_{20}$, siendo muy baja la presencia en los tratamientos $\mathrm{CL}_{30^{\prime}} \mathrm{CL}_{40}$ y $\mathrm{CL}_{50}$. En el caso de los tratamientos tipo LC, la presencia de ésta especie solo se encontró en los tratamientos $\mathrm{LC}_{10}$ y LC ${ }_{30}$ (Jabran et al., 2015).

Los tratamientos en los que se encontró mayor producción de biomasa fueron en el $\mathrm{LC}_{30} \mathrm{y} \mathrm{LC}_{10}$. En $\mathrm{CL}_{40}$ se presentó una mayor diversidad de especies acompañantes al cultivo. En $\mathrm{LC}_{20}$ se observó un menor número de especies. Las especies más frecuentes durante todo el ensayo fueron falso poleo (Drymarya cordata L.), R. acetocella L., S. arvensis L. y S. vulgaris L. Las especies rábano (Brasica rapa L.) y el Ray grass (Lolium sp.) fueron de menor frecuencia (Gaba et al., 2014).
Respecto a producción de biomasa, las especies que mostraron menores valores fueron $S$. vulgaris y $S$. arvensis, probablemente debido a que son anuales, cuyo ciclo tiene una duración inferior en tiempo a de la especie cultivada, por lo que su presencia se observa principalmente en las fases iníciales del cultivo (Gantoli et al., 2013). Villegas et al. (2004) en un estudio de determinación de periodo crítico de competencia en el cultivo de trigo, hallaron resultados similares relacionados con las mayores producciones de biomasa a medida que se incrementa el tiempo en competencia con el cultivo.

En la Tabla 4, se presenta los resultados de la prueba de Tukey $(\alpha=0,05)$ para la variable de producción total de biomasa en las malezas. El análisis estadístico mostró diferencias significativas entre los tratamientos evaluados, las cuales variaron según el tiempo en el que se mantuvo la competencia con el cultivo, encontrándose mayor producción de biomasa de plantas maleza y menor biodiversidad 
de especies en los tratamientos que se mantuvieron inicialmente libres de maleza y luego en competencia (LC). En éstos tratamientos, las especies que se colectaron en las primeras deshierbas estuvieron principalmente compuestas por $S$. vulgaris, S. arvensis y T. repens.

Tabla 4. Prueba de Tukey para la producción total de biomasa $\left(\mathrm{g} \mathrm{m}^{-2}\right)$ de las malezas en el cultivo de zanahoria bajo diferentes tratamientos de interferencia de malezas.

\begin{tabular}{clcl}
\hline Tratamiento & $\begin{array}{c}\text { Biomasa } \\
\mathbf{g ~ m}^{-2}\end{array}$ & Tratamiento & $\begin{array}{c}\text { Biomasa } \\
\mathbf{g ~ m}^{-2}\end{array}$ \\
\hline $\mathrm{LC}_{10}$ & $2573,3^{\mathrm{f}}$ & $\mathrm{CL}_{10}$ & $2205,81^{\mathrm{de}}$ \\
$\mathrm{LC}_{20}$ & $1047,31^{\mathrm{ab}}$ & $\mathrm{CL}_{20}$ & $2096,2^{\mathrm{cd}}$ \\
$\mathrm{LC}_{30}$ & $2485^{\mathrm{ef}}$ & $\mathrm{CL}_{30}$ & $1973,7^{\mathrm{cd}}$ \\
$\mathrm{LC}_{40}$ & $1859,2^{\mathrm{c}}$ & $\mathrm{CL}_{40}$ & $1238,43^{\mathrm{ab}}$ \\
$\mathrm{LC}_{50}$ & $2078,1^{\mathrm{cd}}$ & $\mathrm{CL}_{50}$ & $929^{\mathrm{a}}$ \\
$\mathrm{LTC}$ & & $\mathrm{CTC}$ & $1335,5^{\mathrm{b}}$ \\
\hline
\end{tabular}

Los superíndices (a,b,d,d,f,f) nivel de diferencia estadística entre tratamientos según prueba de Tukey $\alpha=0,05$.

La tendencia previamente mencionada, fue similar a la observada por Bedmar et al. (1999) en un estudio sobre competencia en maíz, en el cual encontraron mayor cantidad de biomasa producida en los tratamientos en los cuales se permite la presencia de malezas desde los inicios del cultivo, situación, que puede ser consecuencia del hábito reproductivo de las especies reportadas, pues, en el caso de $S$. vulgaris, $S$. arvensis y T. repens, son especies foráneas que se caracterizan por ser altamente productoras de estructuras sexuales y asexuales (López, 2010) y que corresponden al habito reproductivo de una especie exótica, que invade un espacio nuevo, en detrimento de las especies nativas (Lodge et al., 2006). Esto estaría ligado a factores representativos de las habilidades competitivas de las malezas, los cuales contribuyen a fortalecer su alta capacidad de persistencia en el medio (Aramendiz et al., 2010).

Durante el ensayo, se encontraron 15 plantas nocivas, de las cuales 13 fueron dicotiledóneas (Üstüner, 2017). En estas especies se concentra más del $90 \%$ de la producción total de biomasa. Estos resultados muestran que las malezas se adaptan mejor a las especies que son similares a ellas, y en este caso, las especies que están en competencia con el cultivo se ven favorecidas por tener al menos una característica en común, frente a un cultivo sensible a las malezas como es $D$. carota (Walsh et al., 2013).

Resultados similares han sido reportados en otros estudios, como los realizados por Aramendiz et al. (2010), en un ensayo de época crítica de competencia en el cultivo de Solanum melongena, y Blanco y Leiva (2011) en un ensayo de determinación de época critica de competencia en el cultivo de fríjol.

Densidad final de zanahoria. Para el caso de la planta cultivada, el mayor número de plantas por metro cuadrado se observó en los tratamientos que se expusieron a competencia y luego permanecieron libres (CL) (Tabla 5). En los tratamientos LTC y LC ${ }_{50}$, se encontraron 42 y 46 plantas $\mathrm{m}^{-2}$, seguidos de los tratamientos $\mathrm{CL}_{10}$ y $\mathrm{CL}_{20}$, con una densidad de 38 y 37 plantas $\mathrm{m}^{-2}$, respectivamente. Los tratamientos que se encontraron en condiciones de competencia total durante todo el ciclo del cultivo (CTC) y en competencia durante los primeros 50 días, tuvieron el menor número de plantas $\mathrm{m}^{-2}$. 
Tabla 5. Plantas de zanahoria, $\mathrm{m}^{-2}$ discriminado por calidad bajo diferentes tratamientos de interferencia de malezas.

\begin{tabular}{lcccc}
\hline Tratamiento & Primera & Tercera & Descarte & Total \\
\hline $\mathrm{CL}_{10}$ & 18 & 13 & 7 & 38 \\
$\mathrm{CL}_{20}$ & 21 & 12 & 5 & 37 \\
$\mathrm{CL}_{30}$ & 15 & 17 & 3 & 35 \\
$\mathrm{CL}_{40}$ & 18 & 13 & 3 & 34 \\
$\mathrm{CL}_{50}$ & 11 & 10 & 8 & 28 \\
$\mathrm{CTC}$ & 4 & 10 & 5 & 19 \\
$\mathrm{LC}$ & 1 & 16 & 4 & 20 \\
$\mathrm{LC}_{20}$ & 11 & 11 & 2 & 24 \\
$\mathrm{LC}_{30}$ & 19 & 9 & 4 & 32 \\
$\mathrm{LC}_{40}$ & 21 & 13 & 3 & 36 \\
$\mathrm{LC}$ & 20 & 13 & 9 & 42 \\
$\mathrm{LCT}$ & 22 & 18 & 6 & 45 \\
\hline
\end{tabular}

En los tratamientos tipo LC, se encontró una densidad de plantas $\mathrm{m}^{-2}$ mayor a las observadas en los tratamientos en competencia desde el inicio del cultivo. Las mayores densidades de plantas $\mathrm{m}^{-2} \mathrm{se}$ observaron en el tratamiento $\mathrm{LC}_{50}$, correspondiente al tratamiento libre de competencia durante los primeros 50 días después de emergido el cultivo, con 41,5 plantas $\mathrm{m}^{-2} \mathrm{y}$ en el tratamiento que se encontró libre de competencia durante todo el ciclo de cultivo (LTC). Las menores densidades se registraron en los tratamientos $\mathrm{LC}_{10}$ y LC $20^{\prime}$ los cuales se mantuvieron libres de competencia los primeros 10 y 20 días del cultivo. En la Tabla 6, se muestra la prueba de Tukey $(\alpha=0,05)$ para la producción de raíces de zanahoria, donde las relaciones de los datos reportados muestran como la producción total fue favorecida cuando los tratamientos estuvieron libres de competencia desde el inicio del cultivo (Knezevic et al., 2015).
Tabla 6. Efecto del tiempo de deshierba sobre el rendimiento del cultivo de zanahoria.

\begin{tabular}{cccc}
\hline Tratamiento & $\begin{array}{c}\text { Rendimiento } \\
\mathbf{g ~ m}^{2}\end{array}$ & Tratamiento & $\begin{array}{c}\text { Rendimiento } \\
\mathbf{g ~ m}^{2}\end{array}$ \\
\hline $\mathrm{LC}_{10}$ & $918,75^{\mathrm{a}}$ & $\mathrm{CL}_{10}$ & $4086,25^{\mathrm{ef}}$ \\
$\mathrm{LC}_{20}$ & $1932,5^{\mathrm{b}}$ & $\mathrm{CL}_{20}$ & $4101,25^{\mathrm{ef}}$ \\
$\mathrm{LC}_{30}$ & $3840^{\text {def }}$ & $\mathrm{CL}_{30}$ & $3276^{\text {cde }}$ \\
$\mathrm{LC}_{40}$ & $4327,5^{\mathrm{f}}$ & $\mathrm{CL}_{40}$ & $3103,7^{\mathrm{cd}}$ \\
$\mathrm{LC}_{50}$ & $5310^{\mathrm{g}}$ & $\mathrm{CL}_{50}$ & $2725^{\mathrm{bc}}$ \\
$\mathrm{LTC}$ & $5810,5^{\mathrm{g}}$ & $\mathrm{CTC}$ & $986,75^{\mathrm{a}}$ \\
\hline
\end{tabular}

Los superíndices ${ }^{(\mathrm{a}, \mathrm{b}, \mathrm{c}, \mathrm{d}, \mathrm{e}, \mathrm{f}, \mathrm{g})}$ nivel de diferencia estadística entre tratamientos según prueba de Tukey $\alpha=0,05$.

La menor producción se registró en los tratamientos CTC con un rendimiento total de $986 \mathrm{~g} \mathrm{~m}^{-2}$ y LC $_{10}$ con de $918,75 \mathrm{~g} \mathrm{~m}^{-2}$, seguidos por $\mathrm{CL}_{50}$ y LC $\mathrm{L}^{\prime}$, con rendimientos de $2.725 \mathrm{~g} \mathrm{~m}^{-2}$ y $1.932,5 \mathrm{~g} \mathrm{~m}^{-2}$, en su orden. Las mayores producciones se obtuvieron en los tratamientos $\mathrm{CL}_{10} \mathrm{y} \mathrm{CL}_{20}$ con rendimientos de $4.086,25 \mathrm{~g}$ $\mathrm{m}^{-2}$ y $4.101,25 \mathrm{~g} \mathrm{~m}^{-2}$, respectivamente. Para los tratamientos LTC, los mayores rendimientos se obtuvieron con $\mathrm{LC}_{50}\left(5.310 \mathrm{~g} \mathrm{~m}^{-2}\right)$ y LC $40\left(4.327,5 \mathrm{~g} \mathrm{~m}^{-2}\right)$.

Los resultados obtenidos mostraron un efecto adverso en la producción, proporcional a la cantidad de tiempo en que el cultivo estuvo en competencia por las malezas. Resultados similares fueron obtenidos por Villegas et al. (2004), en la determinación del PCC entre las plantas maleza y el cultivo de trigo, reportaron un efecto adverso en la producción cuando el cultivo inicia en competencia con las malezas, y con los encontrados por Ljlal et al. (2011) en el cultivo de ajonjolí, donde concluyen que la competencia ejercida por las plantas nocivas al cultivo, afectan la producción. Ellos reportaron menores rendimientos del cultivo, a medida que aumentaba el tiempo en competencia. En todos los tratamientos, se observaron pérdidas por el efecto de competencia interespecífica, siendo éstas significativamente mayores a 
medida que se incrementa el tiempo en el cual la planta cultivada se encuentra compitiendo.

Cuando las especies acompañantes son retiradas del cultivo, las pérdidas representaron alrededor del 40\% del rendimiento, en relación con la producción obtenida en el tratamiento que estuvo libre de competencia durante todo el cultivo. Este efecto de la competencia entre especies y las pérdidas en el rendimiento de plantas cultivadas ya ha sido descrito en otros ensayos como los reportados por Cousens (2008).

El mayor porcentaje de plantas interferentes con el cultivo, encontradas en el estudio, fueron plantas exóticas y únicamente la especie $D$. cordata se reporta como nativa de la región, situación que se debe monitorear, ya que diversos estudios muestran como las plantas exóticas no sólo son más competitivas con especies nativas y cultivadas, sino que también, se pueden convertir en malezas de difícil control en las zonas invadidas.
En la Figura 1, se observa el efecto de la competencia sobre el rendimiento agronómico, encontrándose las menores producciones en los tratamientos $\mathrm{LC}_{10}, \mathrm{LC}_{20}, \mathrm{CL}_{50}$ y CTC, y los mayores rendimientos en los tratamientos $\mathrm{LC}_{50}\left(5.310 \mathrm{~g} \mathrm{~m}^{-2}\right) \mathrm{y}$ LTC $\left(5810,5 \mathrm{~g} \mathrm{~m}^{-2}\right)$. El punto de intersección entre los dos tipos de tratamientos se ubica alrededor de los 28 días después de emergido el cultivo, con un rendimiento de $3.500 \mathrm{~g} \mathrm{~m}^{-2}$. Se presentaron pérdidas significativas bajo las condiciones experimentales, con reducciones en la producción que oscilaron desde el $43 \%$ hasta el $84 \%$ del total, sin embargo estas fueron menores a las que se presentan en el cultivo cuando se permite competencia por encima de la época crítica.

El punto de intersección entre los tratamientos tipo LC y CL se dío aproximadamente en el día 27, con una densidad aproximada de 30plantas $\mathrm{m}^{-2} \mathrm{y}$ una producción media de $3.500 \mathrm{~g} \mathrm{~m}^{-2}$ (Figura 1), lo que sitúa la época crítica de competencia entre los días 20 y 45, después de la emergencia del cultivo.

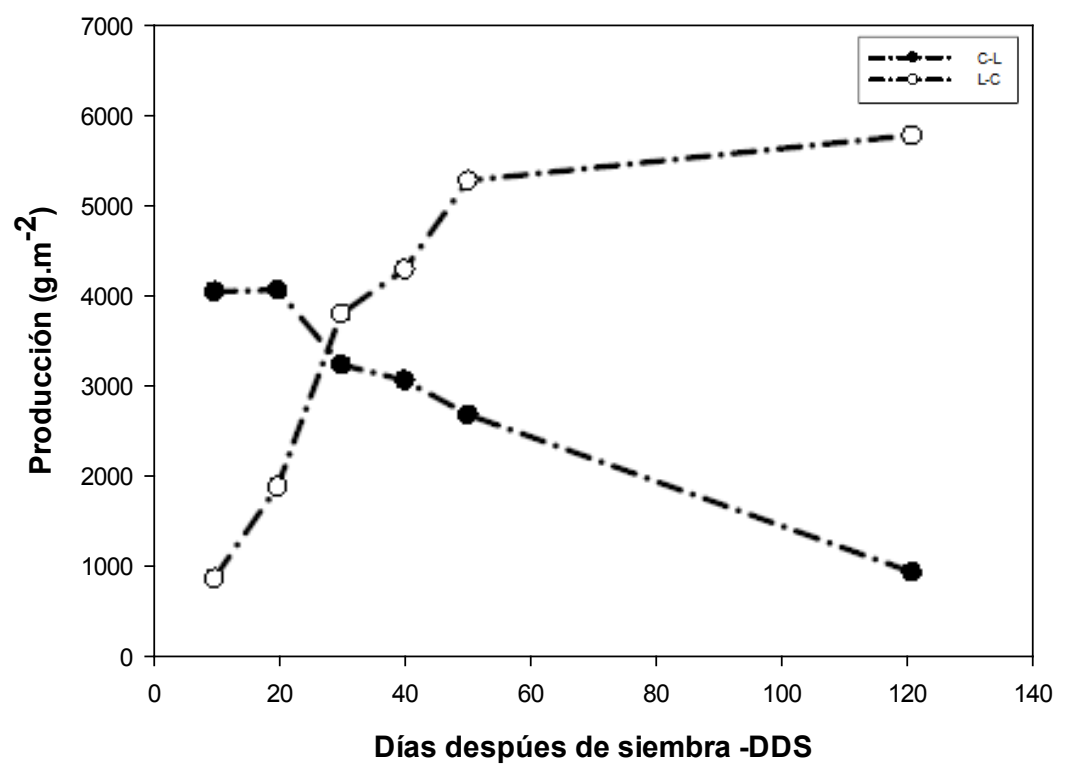

Figura 1. Efecto de la competencia de malezas sobre el rendimiento de zanahoria. 


\section{CONCLUSIONES}

El cultivo de zanahoria es sensible a la competencia durante los primeros 32 días después de la emergencia. El periodo crítico de competencia para el cultivo de Zanahoria se situó entre los 25 y 32 días después de la emergencia.

El mayor rendimiento agronómico se obtuvo en el tratamiento que se mantuvo limpio durante todo el ciclo de cultivo, mostrando una producción de $5.810 \mathrm{~g} \mathrm{~m}^{-2}, 2.252 \mathrm{~g}$ por encima de obtenida en el punto crítico de competencia.

El mayor porcentaje de plantas interferentes con el cultivo, encontradas en el estudio, fueron plantas exóticas y únicamente la especie $D$. cordata se reporta como nativa de la región.

Conflicto de intereses: Los autores declaran que no hay conflicto de interés.

\section{REFERENCIAS BIBLIOGRÁFICAS}

Aramendiz, H., Cardona-Ayala, C. \& Deoro, R. (2010). Periodo de interferencia de arvenses en el cultivo de berenjena (Solanum melongena L.). Agronomia Colombiana. 28(1): 81-88.

Bedmar, F., Manetti, P. \& Monterubbianes, G. (1999). Determination of the critical period of weed control in corn using a thermal basis. Pesquisa Agropecuária Brasileira. 34(2): 188-193.

Blanco, Y. \& Leiva, A. (2011). Determinación del periódo crítico de competencia de las arvenses con el cultivo del frijol (Phaseolus vulgaris L.). Cultivos Tropicales. 32(2): 143-153.

Cheng, D. \& Xu, L. (2015). Predicting the potential distributions of Senecio vulgaris L. in China. Peer Journal Preprints. 3(1). e1612v1. doi: https://doi. org/10.7287/peerj.preprints.1612v1.

Cousens, R. (2008). A simple model relating yield loss to weed density. Annals of Applied Biology. 107 (2): 239-252.
Délye, C., Jasieniuk, M. \& Corre, V. (2013). Deciphering the evolution of herbicide resistance in weeds. Trends in Genetics. 29(11): 649-658.

Gaba, S., Fried, G., Kazakou, E., Chauvel, B. \& Navas, M. L. (2014). Agroecological weed control using a functional approach: a review of cropping systems diversity. Agronomy for Sustainable Development. 34(1): 103-119.

Gantoli, G., Ayala, V. R. \& Gerhards, R. (2013). Determination of the critical period for weed control in corn. Weed Technology. 27(1): 63-71.

Gaviola, J. C. (2013). Manual de producción de zanahoria. Mendoza, Argentina: INTA. 207p.

Holdridge, L. (2000). Ecologia basada en zonas de vida - 5a reimpresión. San José de Costa Rica: IICA. 216p.

Hosseini, P., Karimi, H., Babaei, S., Mashhadi, H. \& Oveisi, M. (2014). Weed seed bank as affected by crop rotation and disturbance. Crop rotation. 64(1): 1-6. doi: https://doi.org/10.1016/j.cropro.2014.05.022.

ICONTEC - Instituto Colombiano de Normas Técnicas y Certificación. (1994). Norma Tecnica Colombiana NTC-1226-Zanahoria. Bogotá: Instituto Colombiano de Normas Técnicas y Certificación (ICONTEC). $124 \mathrm{p}$.

Jabran, K., Mahajan, G., Sardana, V. \& Chauhan, B. S. (2015). Allelopathy for weed control in agricultural systems. Crop Protection. 72: 57-65. doi: https:// doi.org/10.1016/j.cropro.2015.03.004.

Knezevic, S. Z. \& Datta, A. (2015). The critical period for weed control: revisiting data analysis. Weed Science. 63(SP1): 188-202. doi: https://doi.org/10.1614/ WS-D-14-00035.1.

Korres, N. E. \& Norsworthy, J. K. (2015). Influence of a rye cover crop on the critical period for weed control in cotton. Weed Science. 63(1): 346-352. doi: https://doi.org/10.1614/WS-D-14-00075.1.

Landesmann, J. B., Gundel, P. E., Martínez-Ghersa, M. A. \& Ghersa, C. M. (2013). Ozone exposure of a weed community produces adaptive changes in seed populations of Spergula arvensis. PloS one. 8(9): e75820. 
Ljlal, Z., Tanveer, M., Safdar, A., Aziz, M., Ashraf, F., Atif, A. \& Maqbool, M. (2011). Effects of weed crop competition period on weeds and yield and yield components of sesame (Sesamum indicum L.). Pakistan Journal of Weed Science Research. 17: (1): 51-63.

Lodge, D., Stein, R., Brown, K., Covich, A., Bronmark, C., Garvey, J. \& Klosiewski, S. (2006). Predicting impact of freshwater exotic species on native biodiversity challenges in spatial scaling. Australian Journal of Ecology. 23(19): 53-67.

López, G. (2010). Sobre el género Spergula L. (Incl. Spergularia (Pers) Pers. ex j. Presl \& C. Presl, nom cons.) (Cariophyllaceae) y sus especies en la peninsula iberica e islas baleares. Lagascalia. 30(1): 7-18.

Nichols, S. N., Hofmann, R. W. \& Williams, W. M. (2014). Drought resistance of Trifolium repens $\times$ Trifolium uniflorum interspecific hybrids. Crop and Pasture Science. 65(9): 911-921.

Nkuété, A. H., Migliolo, L., Wabo, H. K. \& Tane, P.; Franco, O. L. (2015). Evaluation of multiple functions of Polygonum genus compounds. European Journal of Medicinal Plants. 6(1): 1-16.

Nono, N. R., Nzowa, K. L., Barboni, L. \& Tapondjou, A. L. (2014). Drymaria cordata (Linn.) Willd (Caryophyllaceae): Ethnobotany, pharmacology and phytochemistry. Advances in Biological Chemistry. 4(2): 160-167.

Radosevich, S. R., Holt, J. S. \& Ghersa, C. M. (2007). Ecology of weeds and invasive plants: relationship to agriculture and natural resource management. New Yersey: John Wiley \& Sons. 127p.

Üstüner, T. (2017). Determination of the frequency and density of weed species in apple orchards in Kahramanmaras region of Turkey. Bangladesh Journal of Agricultural Research. 42(1): 87-102.

Villegas, M., Martínez-Díaz, G., Cinco-Castro, R. \& Avendaño, L. (2004). Périodo crítico de competencia de malezas en trigo. Agricultura Técnica en México. 30(2): 223-234.

Walsh, M., Newman, P. \& Powles, S. (2013). Targeting weed seeds in-crop: a new weed control paradigm for global agriculture. Weed Technology. 27(3): 431-436. doi: https://doi.org/10.1614/WT-D-1200181.1 .
Yazdi, S. A. F., Rezvani, M., Mohassel, M. H. R. \& Ghanizadeh, H. (2013). Factors affecting seed germination and seedling emergence of sheep sorrel (Rumex acetosella). Romanian Agricultural Research. 30(1): 373-380. 